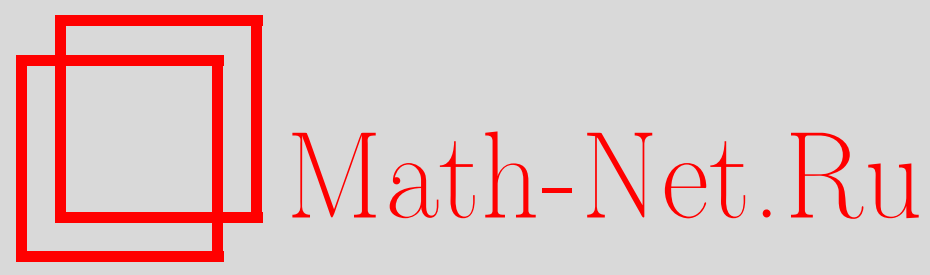

Ю. К. Демьянович, Вложенные пространства тригонометрических сплайнов и их всплесковое разложение, Матем. заметки, 2005, том 78, выпуск 5, 658-675

DOI: https://doi.org/10.4213/mzm2630

Использование Общероссийского математического портала Math-Net.Ru подразумевает, что вы прочитали и согласны с пользовательским соглашением http://www . mathnet.ru/rus/agreement

Параметры загрузки:

IP : 54.92 .164 .108

26 апреля 2023 г., 15:59:19

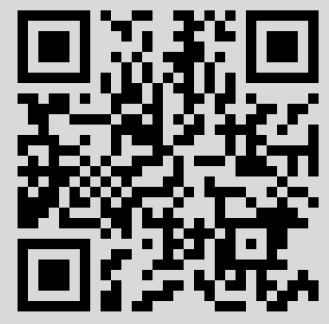




\section{ВЛОЖЕННЫЕ ПРОСТРАНСТВА ТРИГОНОМЕТРИЧЕСКИХ СПЛАЙНОВ И ИХ ВСПЛЕСКОВОЕ РАЗЛОЖЕНИЕ}

Ю.К. Демьянович

С каждой бесконечной сеткой $X: \cdots<x_{-1}<x_{0}<x_{1}<\cdots$ связываются система тригонометрических сплайнов $\left\{\mathfrak{T}_{j}^{B}\right\}$ класса $C^{1}(\alpha, \beta)$, линейное пространство $\mathscr{T}^{B}(X) \stackrel{\text { def }}{=}\left\{\tilde{u} \mid \tilde{u}=\sum_{j} c_{j} \mathfrak{T}_{j}^{B} \forall c_{j} \in \mathbb{R}^{1}\right\}$, функционалы $g^{(i)} \in\left(C^{1}(\alpha, \beta)\right)^{*}$ со свойством биортогональности: $\left\langle g^{(i)}, \mathfrak{T}_{j}^{B}\right\rangle=\delta_{i, j}$ (здесь $\alpha \stackrel{\text { def }}{=} \lim _{j \rightarrow-\infty} x_{j}, \beta \stackrel{\text { def }}{=}$ $\lim _{j \rightarrow+\infty} x_{j}$ ). Для вложенных сеток $\bar{X} \subset X$ устанавливается вложенность соответствуюших пространств $\mathscr{T}^{B}(\bar{X}) \subset \mathscr{T}^{B}(X)$ и выводятся формулы декомпозиции и реконструкции для сплайн-всплескового разложения $\mathscr{T}^{B}(X)=\mathscr{T}^{B}(\bar{X}) \dot{+} W$, полученного с помощью системы упомянутых функционалов.

Библиография: 7 названий.

Известно, что на двукратно измельчающейся сетке $B$-сплайны образуют систему вложенных пространств, на основе которой строятся сплайн-всплесковые разложения (см. [1]-[5]). Применение неравномерной сетки позволяет улучшить приближение функций без усложнения вычислений, а для этого могут понадобиться различные степени измельчения сетки в разных частях рассматриваемого промежутка. Таким образом, рассмотрение сплайн-всплесковых разложений при произвольном измельчении сетки актуально.

Цель данной работы - получить системы вложенных пространств тригонометрических сплайнов, дать их вейвлетное разложение и вывести соответствующие формулы декомпозиции и реконструкции при произвольном способе измельчения сетки.

Для построения многосеточных методов решения эллиптических задач (см. [6]) и для всплесковых разложений при обработке сигналов (см. [1]) удобны вложенные линейные пространства $V_{j}$, лежащие в том или ином функциональном пространстве: $V_{0} \subset V_{1} \subset \cdots \subset V_{j} \subset V_{j+1} \subset \cdots$. При этом строится (явно или неявно) прямое разложение $V_{j+1}=V_{j} \dot{+} W_{j}$, приводящее к представлению $\bigcup_{j=0}^{+\infty} V_{j}=V_{0} \dot{+} W_{0} \dot{+} W_{1} \dot{+} W_{2} \dot{+} \cdots$. Эти построения в $L_{2}\left(\mathbb{R}^{1}\right)$ (через $\mathbb{R}^{1}$ обозначаем вещественную ось) часто связывают с двукратным измельчением равномерной сетки в $\mathbb{R}^{1}$, с задачей отыскания функции $\varphi(t)$ из уравнения $\varphi(t)=\sum_{j} c_{j} \varphi(2 t-j$ ) (назьваемого масштабирующим) и с задачей построения ортогонального разложения $V_{0} \oplus W_{0} \oplus W_{1} \oplus W_{2} \oplus \cdots$; при решении этих задач используется преобразование Фурье (см. [1], [2]). Применение

Работа выполнена при финансовой поддержке Российского фонда фундаментальных исследований, гранты № 04-01-00692 и № 04-01-00026. 
неравномерных сеток позволяет улучшить приближение функций без усложнения вычислений, но для построения сплайн-всплескового разложения масштабирующее уравнение и преобразование Фурье оказьваются недостаточными. Первое заменяется соотношением $\varphi_{i(s)}(t)=\sum_{j} d_{i, j}^{(s)} \varphi_{j(s+1)}(t)$ (назьваемым калибровочным соотношением) между функциями $\varphi_{i(s)} \in V_{s}$, а вместо второго используется биортогональная (к системе функций $\left.\left\{\varphi_{j(s+1)}\right\}\right)$ система функционалов. В случае пространств $B$-сплайнов на последовательности неравномерных двукратно измельчающихся (произвольньпм образом) сеток калибровочные соотношения получаются с помощью специального дробно-рационального тождества (см. [4]), а упомянутое ортогональное разложение заменяется прямым разложением с использованием простого продолжения биортогональной системы функционалов (см. [5]). Однако для дальнейшего улучшения приближения функций (например, функций с особенностью) даже произвольное двукратное измельчение сетки недостаточно: важно иметь возможность добавлять произвольное (конечное) число новых узлов между любой парой соседних узлов исходной сетки.

В данной работе с бесконечной (вообще говоря неравномерной) сеткой $X$ на открытом интервале $(\alpha, \beta)$ вещественной оси связьваются система тригонометрических сплайнов $\left\{\mathfrak{T}_{j}^{B}\right\}$ класса $C^{1}(\alpha, \beta)$, линейное пространство $\mathscr{T}^{B}(X) \stackrel{\text { def }}{=}\left\{\tilde{u} \mid \tilde{u}=\sum_{j} c_{j} \mathfrak{T}_{j}^{B}\right.$ $\left.\forall c_{j} \in \mathbb{R}^{1}\right\}$ и система функционалов, биортогональная системе $\left\{\mathfrak{T}_{j}^{B}\right\}$. Сначала рассматривается сетка $\bar{X}_{1}$, которая получена из исходной сетки $X$ удалением одного узла, и устанавливаются вложенность соответствующих пространств $\mathscr{T}^{B}\left(\bar{X}_{1}\right) \subset \mathscr{T}^{B}(X)$, а также вьводятся формулы декомпозиции и реконструкции для сплайн-всплескового разложения $\mathscr{T}^{B}(X)=\mathscr{T}^{B}\left(\bar{X}_{1}\right)+W$. Отсюда получается вложенность пространств $\mathscr{T}^{B}(\bar{X}) \subset \mathscr{T}^{B}(X)$ и в том случае, когда сетка $X$ получена добавлением конечного числа новых узлов между любыми соседними узлами исходной сетки $\bar{X}$, а упомянутая выше биортогональная система функционалов приводит к алгоритмам построения калибровочных соотношений и формул декомпозиции/реконструкции соответствующего сплайн-всплескового разложения.

Работа состоит из восьми пунктов. В первом, во втором и в третьем пунктах вводятся необходимые обозначения для сеток, рассматриваемых тригонометрических сплайнов и биортогональных систем. В четвертом пункте устанавливаются две вспомогательные леммы, а в пятом строятся калибровочные соотношения. Остальные пункты посвящены сплайн-всплесковому разложению и соответствующим формулам декомпозиции и реконструкции.

1. Некоторые обозначения. На конечном или бесконечном интервале $(\alpha, \beta)$ вещественной оси $\mathbb{R}^{1}$ рассмотрим сетку

$$
\begin{aligned}
X & \stackrel{\text { def }}{=}\left\{x_{j}\right\}_{j \in \mathbb{Z}}, \quad X: \cdots<x_{-1}<x_{0}<x_{1}<\cdots, \\
\text { где } \alpha & =\lim _{j \rightarrow-\infty} x_{j}, \quad \beta=\lim _{j \rightarrow+\infty} x_{j}, \quad x_{j+1}-x_{j}<\frac{\pi}{2} \quad \forall j \in \mathbb{Z},
\end{aligned}
$$

a $\mathbb{Z}$ - множество всех целых чисел. Символом $C^{1}(\alpha, \beta)$ обозначим линейное пространство функций, непрерьвно дифференцируемых в точках открытого интервала $(\alpha, \beta)$. 
Рассмотрим тригонометрические сплайны вида

$$
\begin{aligned}
\mathfrak{T}_{j}^{B}(t)= & \sin ^{2}\left(t-x_{j}\right) \sin ^{-1}\left(x_{j+2}-x_{j}\right) \sin ^{-1}\left(x_{j+1}-x_{j}\right) \quad \text { для } \quad t \in\left[x_{j}, x_{j+1}\right), \\
\mathfrak{T}_{j}^{B}(t)= & \frac{1}{2}\left[\sin \left(x_{j+2}+x_{j+3}-2 t\right) \cos \left(x_{j+1}-x_{j}\right)+\sin \left(2 t-x_{j}-x_{j+1}\right)\right. \\
& \left.\times \cos \left(x_{j+3}-x_{j+2}\right)+\sin \left(x_{j+1}+x_{j}-x_{j+2}-x_{j+3}\right)\right] \sin ^{-1}\left(x_{j+2}-x_{j+1}\right) \\
& \quad \times \sin ^{-1}\left(x_{j+3}-x_{j+1}\right) \sin ^{-1}\left(x_{j+2}-x_{j}\right) \quad \text { для } \quad t \in\left[x_{j+1}, x_{j+2}\right), \\
\mathfrak{T}_{j}^{B}(t)= & \sin ^{2}\left(t-x_{j+3}\right) \sin ^{-1}\left(x_{j+3}-x_{j+1}\right) \sin ^{-1}\left(x_{j+3}-x_{j+2}\right) \quad \text { для } t \in\left[x_{j+2}, x_{j+3}\right],
\end{aligned}
$$

причем для остальных $t$ функция $\mathfrak{T}_{j}^{B}$ равна нулю:

$$
\mathfrak{T}_{j}^{B}(t)=0 \quad \forall t \notin\left[x_{j}, x_{j+3}\right], \quad \text { и значит, } \quad \operatorname{supp} \mathfrak{T}_{j}^{B}=\left[x_{j}, x_{j+3}\right] .
$$

Легко проверить, что функция $\mathfrak{T}_{j}^{B}(t)$, задаваемая формулами $(1.3)-(1.6)$, непрерьвно дифференцируема на вещественной оси, вогнута на интервалах $\left(x_{j}, x_{j+1}\right)$ и $\left(x_{j+2}, x_{j+3}\right)$, выпукла на интервале $\left(x_{j+1}, x_{j+2}\right)$, положительна на интервале $\left(x_{j}, x_{j+3}\right)$, а ее вторая производная имеет неустранимые разрывы первого рода в точках $x_{j}, x_{j+1}, x_{j+2}, x_{j+3}$. Эти свойства аналогичны свойствам известного (см., например, $[7$, с. 23]) $B$-сплайна второй степени с носителем $\left[x_{j}, x_{j+3}\right]$. Сплайны $\mathfrak{T}_{j}^{B}(t)$ будем называть $B_{c s}-$ cnлайнами.

ЗАмЕчАниЕ 1. В дальнейшем часто используются следующие формулы:

$$
\begin{gathered}
\mathfrak{T}_{j}^{B}\left(x_{i}\right)=\lim _{t \rightarrow x_{i}-0} \mathfrak{T}_{j}^{B}(t), \quad \mathfrak{T}_{j}^{B}\left(x_{i}\right)=\lim _{t \rightarrow x_{i}+0} \mathfrak{T}_{j}^{B}(t), \\
\left(\mathfrak{T}_{j}^{B}\right)^{\prime}\left(x_{i}\right)=\lim _{t \rightarrow x_{i}-0}\left(\mathfrak{T}_{j}^{B}\right)^{\prime}(t), \quad\left(\mathfrak{T}_{j}^{B}\right)^{\prime}\left(x_{i}\right)=\lim _{t \rightarrow x_{i}+0}\left(\mathfrak{T}_{j}^{B}\right)^{\prime}(t), \\
\mathfrak{T}_{j}^{B}\left(x_{j}\right)=0, \quad\left(\mathfrak{T}_{j}^{B}\right)^{\prime}\left(x_{j}\right)=0, \quad \mathfrak{T}_{j}^{B}\left(x_{j+3}\right)=0, \quad\left(\mathfrak{T}_{j}^{B}\right)^{\prime}\left(x_{j+3}\right)=0 .
\end{gathered}
$$

Эти формулы справедливы благодаря импликации $\mathfrak{T}_{j} \in C^{1}(\alpha, \beta)$.

2. Биортогональная система функционалов. В пространстве $C^{1}(\alpha, \beta)$ pacсмотрим линейные функционалы

$$
g^{(i)} \stackrel{\text { def }}{=} \cos \left(x_{i+2}-x_{i+1}\right)+\left.\frac{1}{2} \sin \left(x_{i+2}-x_{i+1}\right) \frac{d}{d t}\right|_{t=x_{i+1}}, \quad i \in \mathbb{Z},
$$

действуюшие на функции $u \in C^{1}(\alpha, \beta)$ по формуле

$$
\left\langle g^{(i)}, u\right\rangle \stackrel{\text { def }}{=} \cos \left(x_{i+2}-x_{i+1}\right) u\left(x_{i+1}\right)+\frac{1}{2} \sin \left(x_{i+2}-x_{i+1}\right) u^{\prime}\left(x_{i+1}\right) .
$$

Из формулы (2.1) видно, что носителем функционала $g^{(i)}$ является точка $x_{i+1}$ :

$$
\operatorname{supp} g^{(i)}=x_{i+1} \text {. }
$$

Теорема 1. Система функиионалов $\left\{g^{(i)}\right\}_{i \in \mathbb{Z}}$ биортогональна системе $B_{c s^{-}}$ сплайнов $\left\{\mathfrak{T}_{j}^{B}\right\}_{j \in \mathbb{Z}}$, m.e. $\left\langle g^{(i)}, \mathfrak{T}_{j}^{B}\right\rangle=\delta_{i, j}$. 
ДокАЗАТЕЛЬСТво. Ввиду замечания 1 при $i=j$ получаем

$$
\begin{aligned}
\left\langle g^{(j)}, \mathfrak{T}_{j}^{B}\right\rangle= & \left\langle\cos \left(x_{j+2}-x_{j+1}\right)+\left.\frac{1}{2} \sin \left(x_{j+2}-x_{j+1}\right) \frac{d}{d t}\right|_{t=x_{j+1}}, \mathfrak{T}_{j}^{B}\right\rangle \\
= & \left\langle\cos \left(x_{j+2}-x_{j+1}\right)+\left.\frac{1}{2} \sin \left(x_{j+2}-x_{j+1}\right) \frac{d}{d t}\right|_{t=x_{j+1}}, \sin ^{2}\left(t-x_{j}\right)\right\rangle \\
& \times \sin ^{-1}\left(x_{j+2}-x_{j}\right) \sin ^{-1}\left(x_{j+1}-x_{j}\right) \\
= & \sin \left(x_{j+1}-x_{j}\right)\left[\sin \left(x_{j+1}-x_{j}\right) \cos \left(x_{j+2}-x_{j+1}\right)\right. \\
& \left.+\cos \left(x_{j+1}-x_{j}\right) \sin \left(x_{j+2}-x_{j+1}\right)\right] \sin ^{-1}\left(x_{j+2}-x_{j}\right) \sin ^{-1}\left(x_{j+1}-x_{j}\right) .
\end{aligned}
$$

Применяя очевидное равенство

$$
\sin \left(x_{j+1}-x_{j}\right) \cos \left(x_{j+2}-x_{j+1}\right)+\cos \left(x_{j+1}-x_{j}\right) \sin \left(x_{j+2}-x_{j+1}\right)=\sin \left(x_{j+2}-x_{j}\right)
$$

из (2.3) имеем

$$
\left\langle g^{(j)}, \mathfrak{T}_{j}^{B}\right\rangle=1 .
$$

При $i=j+1$ с помощью формулы (1.5) находим

$$
\begin{aligned}
\left\langle g^{(j+1)}, \mathfrak{T}_{j}^{B}\right\rangle= & \left\langle\cos \left(x_{j+3}-x_{j+2}\right)+\left.\frac{1}{2} \sin \left(x_{j+3}-x_{j+2}\right) \frac{d}{d t}\right|_{t=x_{j+2}}, \mathfrak{T}_{j}^{B}\right\rangle \\
= & \left\langle\cos \left(x_{j+3}-x_{j+2}\right)+\left.\frac{1}{2} \sin \left(x_{j+3}-x_{j+2}\right) \frac{d}{d t}\right|_{t=x_{j+2}}, \sin ^{2}\left(t-x_{j+3}\right)\right\rangle \\
& \times \sin ^{-1}\left(x_{j+3}-x_{j+1}\right) \sin ^{-1}\left(x_{j+3}-x_{j+2}\right) .
\end{aligned}
$$

Поскольку

$$
\begin{aligned}
& \left\langle\cos \left(x_{j+3}-x_{j+2}\right)+\left.\frac{1}{2} \sin \left(x_{j+3}-x_{j+2}\right) \frac{d}{d t}\right|_{t=x_{j+2}}, \sin ^{2}\left(t-x_{j+3}\right)\right\rangle \\
& =\cos \left(x_{j+3}-x_{j+2}\right) \sin ^{2}\left(x_{j+2}-x_{j+3}\right) \\
& \quad+\sin \left(x_{j+3}-x_{j+2}\right) \sin \left(x_{j+2}-x_{j+3}\right) \cos \left(x_{j+2}-x_{j+3}\right)=0
\end{aligned}
$$

из (2.5) вьводим

$$
\left\langle g^{(j+1)}, \mathfrak{T}_{j}^{B}\right\rangle=0 .
$$

Для $i \leqslant j-1$ и для $i \geqslant j+2$ при подстановке $t=x_{i+1}$ выбираем инцидентный точке $x_{i+1}$ промежуток, где функция $\mathfrak{T}_{j}^{B}(t)$ тож дественно равна нулю, и получаем (см. замечание 1 ) для этих $i$ соотношения

$$
\left\langle g^{(i)}, \mathfrak{T}_{j}^{B}\right\rangle=0 \quad \forall i \in \mathbb{Z} \backslash\{j, j+1\}
$$

Из формул $(2.4),(2.6)$ и (2.7) видно, что теорема полностью доказана. 
3. Переход к новой сетке удалением одного узла. Вводя обозначения

$$
\xi \stackrel{\text { def }}{=} x_{1}, \quad \bar{x}_{j} \stackrel{\text { def }}{=} x_{j} \quad \text { при } j \leqslant 0, \quad \text { и } \quad \bar{x}_{j} \stackrel{\text { def }}{=} x_{j+1} \quad \text { при } j \geqslant 1,
$$

рассмотрим новую сетку $\bar{X}_{1}: \cdots<\bar{x}_{-1}<\bar{x}_{0}<\bar{x}_{1}<\cdots$, полученную из сетки $X$ (см. $(1.1),(1.2))$ удалением узла $x_{1} \cdot B_{c s}$-сплайны $\overline{\mathfrak{T}}_{j}^{B}$, построенные с использованием новой сетки $\bar{X}_{1}$, представляются теми же формулами (1.3)-(1.6) с заменой узлов $x_{j}$ сетки $X$ на узлы $\bar{x}_{j}$ сетки $\bar{X}_{1}$. Для $j \notin\{-2,-1,0\}$ сплайны $\overline{\mathfrak{T}}_{j}^{B}$ совпадают с рассмотренными ранее:

$$
\overline{\mathfrak{T}}_{j}^{B}(t) \equiv \mathfrak{T}_{j}^{B}(t) \quad \forall j \leqslant-3, \quad \overline{\mathfrak{T}}_{j}^{B}(t) \equiv \mathfrak{T}_{j+1}^{B}(t) \quad \forall j \geqslant 1 .
$$

При $j \in\{-2,-1,0\}$ сплайны $\overline{\mathfrak{T}}_{j}^{B}$ могут быть представлены в виде линейной комбинации сплайнов $\mathfrak{T}_{j}^{B}$ и $\mathfrak{T}_{j+1}^{B}$ (см. ниже теорему 2$)$.

По теореме 1 система $\left\{\bar{g}^{(i)}\right\}_{i \in \mathbb{Z}}$ функционалов

$$
\left\langle\bar{g}^{(i)}, u\right\rangle \stackrel{\text { def }}{=} \cos \left(\bar{x}_{i+2}-\bar{x}_{i+1}\right) u\left(\bar{x}_{i+1}\right)+\frac{1}{2} \sin \left(\bar{x}_{i+2}-\bar{x}_{i+1}\right) u^{\prime}\left(\bar{x}_{i+1}\right)
$$

биортогональна системе сплайнов $\left\{\overline{\mathfrak{T}}_{j}^{B}\right\}_{j \in \mathbb{Z} . \text { Видно, что }}$

$$
\bar{g}^{(i)}=g^{(i)} \quad \text { при } \quad i \leqslant-2, \quad \text { и } \quad \bar{g}^{(i)}=g^{(i+1)} \quad \text { при } i \geqslant 0 .
$$

\section{4. Вспомогательные утверждения.}

Лемма 1. Для любых $a, b, c, d, e \in \mathbb{R}^{1}$ справедливо тождество

$$
\begin{aligned}
& {[\sin (c+e) \cos (b-a)-\sin (a+b) \cos (e-c)+\sin (a+b-c-e)] \sin (d-b)} \\
& \quad \equiv[\sin (c+d) \cos (b-a)-\sin (a+b) \cos (d-c)+\sin (a+b-c-d)] \sin (e-b) \\
& \quad+2 \sin (e-d) \sin ^{2} b \sin (c-a) .
\end{aligned}
$$

ДокАЗАТЕЛЬСТво. Выражение в квадратных скобках левой части соотношения (4.1) обозначим $T(e)$,

$$
T(e) \stackrel{\text { def }}{=} \sin (c+e) \cos (b-a)-\sin (a+b) \cos (e-c)+\sin (a+b-c-e) \text {. }
$$

Используя формулу для синуса разности,

$$
\sin (a+b-c-e)=\sin (a+b) \cos (c+e)-\sin (c+e) \cos (a+b),
$$

получаем

$$
\begin{aligned}
T(e) & =\sin (c+e)[\cos (b-a)-\cos (a+b)]+\sin (a+b)[\cos (c+e)-\cos (c-e)] \\
& =\sin (c+e) 2 \sin a \sin b-\sin (a+b) 2 \sin c \sin e \\
& =2[(\sin c \cos e+\sin e \cos c) \sin a \sin b-(\sin a \cos b+\sin b \cos a) \sin c \sin e]
\end{aligned}
$$


откуда имеем

$$
\begin{aligned}
T(e)= & 2[\sin a \sin b \sin c \cos e+\sin a \sin b \cos c \sin e \\
& -\sin a \cos b \sin c \sin e-\cos a \sin b \sin c \sin e] .
\end{aligned}
$$

Тождество (4.1) эквивалентно соотношению

$$
T(e) \sin (d-b)-T(d) \sin (e-b) \stackrel{?}{=} 2 \sin (e-d) \sin ^{2} b \sin (c-a),
$$

где символ ‘竎’ означает, что тождественность левой и правой частей находится в процессе доказательства. Ввиду формулы (4.3) из (4.4) находим

$$
\begin{aligned}
& \sin a \sin b \sin c[\cos e \sin (d-b)-\cos d \sin (e-b)] \\
& \quad+[\sin a \sin b \cos c-\sin a \cos b \sin c-\cos a \sin b \sin c] \\
& \quad \times[\sin e \sin (d-b)-\sin d \sin (e-b)] \\
& \stackrel{?}{=} \sin (e-d) \sin ^{2} b \sin (c-a),
\end{aligned}
$$

откуда, используя в первой и в последней квадратных скобках (для перехода от произведения к сумме тригонометрических функций) формулы

$$
2 \sin \gamma \cos \delta=[\sin (\gamma+\delta)+\sin (\gamma-\delta)], \quad 2 \sin \gamma \sin \delta=[\cos (\gamma-\delta)-\cos (\gamma+\delta)],
$$

соответственно получаем

$$
\begin{aligned}
& {[\sin (-b+d-e)+\sin (b+d-e)] \sin a \sin b \sin c+[\sin a \sin b \cos c-\sin a \cos b \sin c} \\
& \quad-\cos a \sin b \sin c][\cos (b-d+e)-\cos (b+d-e)] \stackrel{?}{=} 2 \sin (e-d) \sin ^{2} b \sin (c-a) .
\end{aligned}
$$

Снова используя формулы (4.5) для перехода от суммы тригонометрических функций к произведению в первой и в последней квадратных скобках соотношения (4.6), находим очевидное тождество

$$
[\sin a \cos c-\cos a \sin c] \sin ^{2} b \sin (d-e) \stackrel{?}{=} \sin (e-d) \sin ^{2} b \sin (c-a) .
$$

Ввиду эквивалентного характера всех переходов из справедливости тождества (4.7) вытекает справедливость тождества (4.1). Лемма доказана.

Лемма 2. Для любых $a, b, c, d \in \mathbb{R}^{1}$ справедливо соотношение

$$
\begin{aligned}
& 2 \sin ^{2} d \sin (c-a) \sin (c-b) \equiv 2 \sin ^{2} c \sin (d-a) \sin (d-b) \\
& \quad+\sin (d-c)[\sin (c+d) \cos (b-a)-\sin (a+b) \cos (d-c)+\sin (a+b-c-d)] .
\end{aligned}
$$


ДокАЗАТЕЛЬСТво. Нетрудно видеть, что в квадратных скобках содержится выражение $T(d)$ (см. формулу (4.2)). Введем обозначения

$$
\begin{aligned}
& A=\sin a, \quad B=\sin b, \quad C=\sin c, \quad D=\sin d, \\
& \bar{A}=\cos a, \quad \bar{B}=\cos b, \quad \bar{C}=\cos c, \quad \bar{D}=\cos d \text {. }
\end{aligned}
$$

Согласно формуле (4.3)

$$
T(d)=2(A B C \bar{D}+A B \bar{C} D-A \bar{B} C D-\bar{A} B C D)
$$

Рассмотрим разность

$$
R \stackrel{\text { def }}{=} \sin ^{2} d \sin (c-a) \sin (c-b)-\sin ^{2} c \sin (d-a) \sin (d-b) .
$$

Используя формулу синуса разности и введенные обозначения $(4.9)$, имеем

$$
R=-A \bar{B} C \bar{C} D^{2}-\bar{A} B C \bar{C} D^{2}+A B \bar{C}^{2} D^{2}+A \bar{B} C^{2} D \bar{D}+\bar{A} B C^{2} D \bar{D}-A B C^{2} \bar{D}^{2} .
$$

Доказьваемоетождество (4.8) эквивалентно соотношению $2 R \stackrel{?}{\equiv} \sin (d-c) T(d)$, которое благодаря формулам $(4.10),(4.11)$ и обозначениям $(4.9)$ можно переписать в виде

$$
\begin{aligned}
& -A \bar{B} C \bar{C} D^{2}-\bar{A} B C \bar{C} D^{2}+A B \bar{C}^{2} D^{2}+A \bar{B} C^{2} D \bar{D}+\bar{A} B C^{2} D \bar{D}-A B C^{2} \bar{D}^{2} \\
& \quad \stackrel{?}{=}(\bar{C} D-C \bar{D})(A B C \bar{D}+A B \bar{C} D-A \bar{B} C D-\bar{A} B C D) .
\end{aligned}
$$

Справедливость последнего соотношения становится очевидной после раскрытия скобок и приведения подобных членов. Рассмотренные преобразования обратимы и, значит, тождество (4.8) справедливо. Лемма доказана.

\section{5. Калибровочные соотношения для $B_{c s}$-сплайнов.}

ТеОрема 2. При $j=-2,-1,0$ функиии $\overline{\mathfrak{T}}_{j}^{B}$ выражсаются через функиии $\mathfrak{T}_{i}^{B}$ по следующим формулам:

$$
\begin{gathered}
\overline{\mathfrak{T}}_{-2}^{B}(t)=\mathfrak{T}_{-2}^{B}(t)+\sin \left(\bar{x}_{1}-\xi\right) \sin ^{-1}\left(\bar{x}_{1}-\bar{x}_{-1}\right) \mathfrak{T}_{-1}^{B}(t), \\
\overline{\mathfrak{T}}_{-1}^{B}(t)=\sin \left(\xi-\bar{x}_{-1}\right) \sin ^{-1}\left(\bar{x}_{1}-\bar{x}_{-1}\right) \mathfrak{T}_{-1}^{B}(t)+\sin \left(\bar{x}_{2}-\xi\right) \sin ^{-1}\left(\bar{x}_{2}-\bar{x}_{0}\right) \mathfrak{T}_{0}^{B}(t) \\
\overline{\mathfrak{T}}_{0}^{B}(t)=\sin \left(\xi-\bar{x}_{0}\right) \sin ^{-1}\left(\bar{x}_{2}-\bar{x}_{0}\right) \mathfrak{T}_{0}^{B}(t)+\mathfrak{T}_{1}^{B}(t) .
\end{gathered}
$$


ДокАЗАТЕльство. Общий план доказательства состоит в следующем: сначала будет доказано тождество (5.1), затем тождество (5.3) сведением его к тождеству (5.1); последним будет доказано тождество (5.2).

1. Начиная доказательство тождества (5.1), заметим, что его достаточно доказать на промежутках $\left[x_{-2}, x_{-1}\right],\left[x_{-1}, x_{0}\right],\left[x_{0}, x_{1}\right],\left[x_{1}, x_{2}\right]$. Последовательно рассмотрим каждый из этих промежутков.

1.1. Пусть $t \in\left[x_{-2}, x_{-1}\right]$. Тогда левая часть соотношения (5.1) определяется формулой (1.3), где $j=-2, x_{i}=\bar{x}_{i}, i=-2,-1,0$ :

$$
\overline{\mathfrak{T}}_{-2}^{B}(t)=\sin ^{2}\left(t-\bar{x}_{-2}\right) \sin ^{-1}\left(\bar{x}_{0}-\bar{x}_{-2}\right) \sin ^{-1}\left(\bar{x}_{-1}-\bar{x}_{-2}\right) .
$$

Для первого слагаемого правой части соотношения (5.1) на промежутке $t \in\left[x_{-2}, x_{-1}\right]$ справедлива формула (1.3); ее перепишем с учетом равенств (3.1):

$$
\mathfrak{T}_{-2}^{B}(t)=\sin ^{2}\left(t-\bar{x}_{-2}\right) \sin ^{-1}\left(\bar{x}_{0}-\bar{x}_{-2}\right) \sin ^{-1}\left(\bar{x}_{-1}-\bar{x}_{-2}\right) .
$$

Второе слагаемое правой части формулы (5.1) при рассматриваемых $t$ равно нулю, а формулы (5.4), (5.5) дают тождество $\overline{\mathfrak{T}}_{-2}^{B}(t) \equiv \mathfrak{T}_{-2}^{B}(t) \forall t \in\left[x_{-2}, x_{-1}\right]$; итак, соотношение (5.1) при $t \in\left[x_{-2}, x_{-1}\right]$ доказано.

1.2. Пусть $t \in\left[x_{-1}, x_{0}\right]$. Тогда левая часть соотношения (5.1) задается формулой (1.4), в которой $j=-2, x_{i}=\bar{x}_{i}, i=-2,-1,0,1$ :

$$
\begin{aligned}
& \overline{\mathfrak{T}}_{-2}^{B}(t)=\frac{1}{2}\left[\sin \left(\bar{x}_{0}+\bar{x}_{1}-2 t\right) \cos \left(\bar{x}_{-1}-\bar{x}_{-2}\right)+\sin \left(2 t-\bar{x}_{-2}-\bar{x}_{-1}\right) \cos \left(\bar{x}_{1}-\bar{x}_{0}\right)\right. \\
& \left.\quad+\sin \left(\bar{x}_{-1}+\bar{x}_{-2}-\bar{x}_{0}-\bar{x}_{1}\right)\right] \sin ^{-1}\left(\bar{x}_{0}-\bar{x}_{-1}\right) \sin ^{-1}\left(\bar{x}_{1}-\bar{x}_{-1}\right) \sin ^{-1}\left(\bar{x}_{0}-\bar{x}_{-2}\right) .
\end{aligned}
$$

Первое слагаемое правой части вычисляется по формуле (1.4) при $j=-2$; перейдем в нем к обозначениям (3.1):

$$
\begin{aligned}
& \mathfrak{T}_{-2}^{B}(t)=\frac{1}{2}\left[\sin \left(\bar{x}_{0}+\xi-2 t\right) \cos \left(\bar{x}_{-1}-\bar{x}_{-2}\right)+\sin \left(2 t-\bar{x}_{-2}-\bar{x}_{-1}\right) \cos \left(\xi-\bar{x}_{0}\right)\right. \\
& \left.\quad+\sin \left(\bar{x}_{-1}+\bar{x}_{-2}-\bar{x}_{0}-\xi\right)\right] \sin ^{-1}\left(\bar{x}_{0}-\bar{x}_{-1}\right) \sin ^{-1}\left(\xi-\bar{x}_{-1}\right) \sin ^{-1}\left(\bar{x}_{0}-\bar{x}_{-2}\right) .
\end{aligned}
$$

Второе слагаемое правой части соотношения (5.1) вычисляется по формуле (1.3) при $j=-1$; перейдем здесь тоже к обозначениям (3.1):

$$
\mathfrak{T}_{-1}^{B}(t)=\sin ^{2}\left(t-\bar{x}_{-1}\right) \sin ^{-1}\left(\xi-\bar{x}_{-1}\right) \sin ^{-1}\left(\bar{x}_{0}-\bar{x}_{-1}\right) .
$$

В соответствии с (5.1) требуется доказать тождество

$$
\begin{aligned}
& {\left[\sin \left(\bar{x}_{0}+\bar{x}_{1}-2 t\right) \cos \left(\bar{x}_{-1}-\bar{x}_{-2}\right)+\sin \left(2 t-\bar{x}_{-2}-\bar{x}_{-1}\right) \cos \left(\bar{x}_{1}-\bar{x}_{0}\right)\right.} \\
& \left.\quad+\sin \left(\bar{x}_{-1}+\bar{x}_{-2}-\bar{x}_{0}-\bar{x}_{1}\right)\right] \sin ^{-1}\left(\bar{x}_{0}-\bar{x}_{-1}\right) \sin ^{-1}\left(\bar{x}_{1}-\bar{x}_{-1}\right) \sin ^{-1}\left(\bar{x}_{0}-\bar{x}_{-2}\right) \\
& \stackrel{?}{\equiv}\left[\sin \left(\bar{x}_{0}+\xi-2 t\right) \cos \left(\bar{x}_{-1}-\bar{x}_{-2}\right)+\sin \left(2 t-\bar{x}_{-2}-\bar{x}_{-1}\right) \cos \left(\xi-\bar{x}_{0}\right)\right. \\
& \left.\quad+\sin \left(\bar{x}_{-1}+\bar{x}_{-2}-\bar{x}_{0}-\xi\right)\right] \sin ^{-1}\left(\bar{x}_{0}-\bar{x}_{-1}\right) \sin ^{-1}\left(\xi-\bar{x}_{-1}\right) \sin ^{-1}\left(\bar{x}_{0}-\bar{x}_{-2}\right) \\
& \quad+2 \sin \left(\bar{x}_{1}-\xi\right) \sin ^{-1}\left(\bar{x}_{1}-\bar{x}_{-1}\right) \sin ^{2}\left(t-\bar{x}_{-1}\right) \sin ^{-1}\left(\xi-\bar{x}_{-1}\right) \sin ^{-1}\left(\bar{x}_{0}-\bar{x}_{-1}\right) .
\end{aligned}
$$


Освобождаясь от знаменателей, видим, что достаточно доказать соотношение

$$
\begin{aligned}
& {\left[\sin \left(\bar{x}_{0}+\bar{x}_{1}-2 t\right) \cos \left(\bar{x}_{-1}-\bar{x}_{-2}\right)+\sin \left(2 t-\bar{x}_{-2}-\bar{x}_{-1}\right) \cos \left(\bar{x}_{1}-\bar{x}_{0}\right)\right.} \\
& \left.\quad+\sin \left(\bar{x}_{-1}+\bar{x}_{-2}-\bar{x}_{0}-\bar{x}_{1}\right)\right] \sin \left(\xi-\bar{x}_{-1}\right) \stackrel{?}{\equiv}\left[\sin \left(\bar{x}_{0}+\xi-2 t\right) \cos \left(\bar{x}_{-1}-\bar{x}_{-2}\right)\right. \\
& \left.\quad+\sin \left(2 t-\bar{x}_{-2}-\bar{x}_{-1}\right) \cos \left(\xi-\bar{x}_{0}\right)+\sin \left(\bar{x}_{-1}+\bar{x}_{-2}-\bar{x}_{0}-\xi\right)\right] \sin \left(\bar{x}_{1}-\bar{x}_{-1}\right) \\
& \quad+2 \sin \left(\bar{x}_{1}-\xi\right) \sin ^{2}\left(t-\bar{x}_{-1}\right) \sin \left(\bar{x}_{0}-\bar{x}_{-2}\right) .
\end{aligned}
$$

Полагая в доказанном ранее тождестве (4.1) (см. лемму 1) $a \stackrel{\text { def }}{=} \bar{x}_{-2}-t, b \stackrel{\text { def }}{=} \bar{x}_{-1}-t$, $c \stackrel{\text { def }}{=} \bar{x}_{0}-t, d \stackrel{\text { def }}{=} \xi-t, e \stackrel{\text { def }}{=} \bar{x}_{1}-t$, приходим к соотношению (5.6). Ввиду обратимости проделанных преобразований заключаем, что тождество (5.1) доказано для $t \in\left[x_{-1}, x_{0}\right]$.

1.3. Рассмотрим теперь промежуток $\left[x_{0}, x_{1}\right]$. На этом промежутке левая часть соотношения (5.1) получается из формулы (1.5) заменами $j=-2, x_{i}=\bar{x}_{i}$ при $i=-1,0,1$ :

$$
\overline{\mathfrak{T}}_{-2}^{B}(t)=\sin ^{2}\left(t-\bar{x}_{1}\right) \sin ^{-1}\left(\bar{x}_{1}-\bar{x}_{-1}\right) \sin ^{-1}\left(\bar{x}_{1}-\bar{x}_{0}\right) \quad \text { для } t \in\left[\bar{x}_{0}, \bar{x}_{1}\right] .
$$

Ту же формулу (1.5) используем и для функции $\mathfrak{T}_{-2}^{B}$, а затем произведем замены обозначений согласно формулам (3.1):

$$
\mathfrak{T}_{-2}^{B}(t)=\sin ^{2}(t-\xi) \sin ^{-1}\left(\xi-\bar{x}_{-1}\right) \sin ^{-1}\left(\xi-\bar{x}_{0}\right) .
$$

Наконец, для функции $\mathfrak{T}_{-1}^{B}$ используем формулу (1.4) при $j=-1$ и сделаем замены по формулам (3.1):

$$
\begin{gathered}
\mathfrak{T}_{-1}^{B}(t)=\frac{1}{2}\left[\sin \left(\xi+\bar{x}_{1}-2 t\right) \cos \left(\bar{x}_{0}-\bar{x}_{-1}\right)+\sin \left(2 t-\bar{x}_{-1}-\bar{x}_{0}\right) \cos \left(\bar{x}_{1}-\xi\right)\right. \\
\left.+\sin \left(\bar{x}_{-1}+\bar{x}_{0}-\xi-\bar{x}_{1}\right)\right] \sin ^{-1}\left(\xi-\bar{x}_{0}\right) \sin ^{-1}\left(\bar{x}_{1}-\bar{x}_{0}\right) \sin ^{-1}\left(\xi-\bar{x}_{-1}\right) .
\end{gathered}
$$

Таким образом, требуется установить тождество

$$
\begin{aligned}
& 2 \sin ^{2}\left(t-\bar{x}_{1}\right) \sin ^{-1}\left(\bar{x}_{1}-\bar{x}_{-1}\right) \sin ^{-1}\left(\bar{x}_{1}-\bar{x}_{0}\right) \\
& \stackrel{?}{\equiv} 2 \sin ^{2}(t-\xi) \sin ^{-1}\left(\xi-\bar{x}_{-1}\right) \sin ^{-1}\left(\xi-\bar{x}_{0}\right) \\
& \quad+\sin \left(\bar{x}_{1}-\xi\right) \sin ^{-1}\left(\bar{x}_{1}-\bar{x}_{-1}\right)\left[\sin \left(\xi+\bar{x}_{1}-2 t\right) \cos \left(\bar{x}_{0}-\bar{x}_{-1}\right)\right. \\
& \left.\quad+\sin \left(2 t-\bar{x}-1-\bar{x}_{0}\right) \cos \left(\bar{x}_{1}-\xi\right)+\sin \left(\bar{x}_{-1}+\bar{x}_{0}-\xi-\bar{x}_{1}\right)\right] \\
& \quad \times \sin ^{-1}\left(\xi-\bar{x}_{0}\right) \sin ^{-1}\left(\bar{x}_{1}-\bar{x}_{0}\right) \sin ^{-1}\left(\xi-\bar{x}_{-1}\right) .
\end{aligned}
$$

Умножая последнее соотношение на произведение

$$
\sin \left(\bar{x}_{1}-\bar{x}_{-1}\right) \sin \left(\bar{x}_{1}-\bar{x}_{0}\right) \sin \left(\xi-\bar{x}_{-1}\right) \sin \left(\xi-\bar{x}_{0}\right)
$$

получим

$$
\begin{aligned}
& 2 \sin ^{2}\left(t-\bar{x}_{1}\right) \sin \left(\xi-\bar{x}_{-1}\right) \sin \left(\xi-\bar{x}_{0}\right) \stackrel{?}{\equiv} 2 \sin ^{2}(t-\xi) \sin \left(\bar{x}_{1}-\bar{x}_{-1}\right) \sin \left(\bar{x}_{1}-\bar{x}_{0}\right) \\
& \quad+\sin \left(\bar{x}_{1}-\xi\right)\left[\sin \left(\xi+\bar{x}_{1}-2 t\right) \cos \left(\bar{x}_{0}-\bar{x}_{-1}\right)+\sin \left(2 t-\bar{x}_{-1}-\bar{x}_{0}\right) \cos \left(\bar{x}_{1}-\xi\right)\right. \\
& \left.\quad+\sin \left(\bar{x}_{-1}+\bar{x}_{0}-\xi-\bar{x}_{1}\right)\right] .
\end{aligned}
$$


Производя замены $a \stackrel{\text { def }}{=} \bar{x}_{-1}-t, b \stackrel{\text { def }}{=} \bar{x}_{0}-t, c \stackrel{\text { def }}{=} \xi-t, d \stackrel{\text { def }}{=} \bar{x}_{1}-t$ в тождестве $(4.8)$, , доказанном в лемме 2 , приходим к соотношению (5.7). Итак, тождество (5.7) установлено, а ввиду эквивалентности проводимых преобразований убеждаемся в справедливости тождества (5.1) на промежутке $\left[x_{0}, x_{1}\right]$.

1.4. Перейдем к последнему промежутку $\left[x_{1}, x_{2}\right]$. На этом промежутке для левой части соотношения (5.1) используем формулу (1.5) при $j=-2, x_{i}=\bar{x}_{i}, i=-1,0,1$ :

$$
\overline{\mathfrak{T}}_{-2}^{B}(t)=\sin ^{2}\left(t-\bar{x}_{1}\right) \sin ^{-1}\left(\bar{x}_{1}-\bar{x}_{-1}\right) \sin ^{-1}\left(\bar{x}_{1}-\bar{x}_{0}\right),
$$

и ту же формулу при $j=-1$ :

$$
\mathfrak{T}_{-1}^{B}(t)=\sin ^{2}\left(t-x_{2}\right) \sin ^{-1}\left(x_{2}-x_{0}\right) \sin ^{-1}\left(x_{2}-x_{1}\right),
$$

откуда, используя обозначения (3.1), находим

$$
\mathfrak{T}_{-1}^{B}(t)=\sin ^{2}\left(t-\bar{x}_{1}\right) \sin ^{-1}\left(\bar{x}_{1}-\bar{x}_{0}\right) \sin ^{-1}\left(\bar{x}_{1}-\xi\right) .
$$

Для доказательства формулы (5.1) на промежутке $\left[x_{1}, x_{2}\right]$ осталось заметить, что на упомянутом промежутке первое слагаемое правой части равно нулю, а второе слагаемое представляется произведением выражения $\sin \left(\bar{x}_{1}-\xi\right) \sin ^{-1}\left(\bar{x}_{1}-\bar{x}_{-1}\right)$ на функцию (5.9), что ведет к выражению (5.8). Тождество (5.1) на промежутке $\left[x_{1}, x_{2}\right]$ доказано. На этом заканчивается доказательство тождества (5.1) для всех $t \in(\alpha, \beta)$.

2. Начиная доказательство тождества (5.3), заметим, что (5.3) отличается от (5.1) лишь обозначениями. Действительно, обозначим $\alpha^{\prime} \stackrel{\text { def }}{=}-\beta, \beta^{\prime} \stackrel{\text { def }}{=}-\alpha$ и введем сетку $X^{\prime} \stackrel{\text { def }}{=}\left\{x_{p}^{\prime}\right\}_{p \in \mathbb{Z}}$ с узлами

$$
x_{p}^{\prime} \stackrel{\text { def }}{=}-x_{-p+2}
$$

на этой сетке рассмотрим функции $\mathfrak{T}_{j}^{\prime}\left(t^{\prime}\right), t^{\prime} \in\left(\alpha^{\prime}, \beta^{\prime}\right)$, построенные согласно соотношениям (1.3)-(1.6) с формальной заменой узлов $x_{j}$ на $x_{j}^{\prime}$. Очевидно, эти функции связаны с функциями, ранее построенньми на сетке $X$, соотношениями

$$
\mathfrak{T}_{p}^{\prime B}\left(t^{\prime}\right)=\mathfrak{T}_{-p-1}^{B}\left(-t^{\prime}\right) \quad \forall t^{\prime} \in\left(\alpha^{\prime}, \beta^{\prime}\right) \quad \forall p \in \mathbb{Z} .
$$

Рассмотрим еще сетку $\bar{X}^{\prime} \stackrel{\text { def }}{=}\left\{\bar{x}_{q}^{\prime}\right\}_{q \in \mathbb{Z}}$ с узлами

$$
\bar{x}_{q}^{\prime} \stackrel{\text { def }}{=}-\bar{x}_{-q+1},
$$

представляющую собой укрупнение сетки $X^{\prime}$ за счет выбрасывания узла $\xi^{\prime} \stackrel{\text { def }}{=} x_{1}^{\prime}$; в соответствии с формулой (5.10) имеем

$$
\xi^{\prime}=x_{1}^{\prime}=-x_{1}=-\xi .
$$

Теперь введем функции ' $\overline{\mathfrak{T}}_{j}^{B}\left(t^{\prime}\right)$, построенные согласно соотношениям (1.3)-(1.5) с формальной заменой узлов $x_{j}$ на $\bar{x}_{j}^{\prime} ;$ ясно, что для таких функций справедливы тождества

$$
\overline{\mathfrak{T}}_{q}^{\prime B}\left(t^{\prime}\right)=\overline{\mathfrak{T}}_{-q-2}^{B}\left(-t^{\prime}\right) \quad \forall t^{\prime} \in\left(\alpha^{\prime}, \beta^{\prime}\right) \quad \forall q \in \mathbb{Z} .
$$


Поскольку из соотношений (5.11)-(5.14) вытекают формулы

$$
\begin{aligned}
\overline{\mathfrak{T}}_{-2}^{B}\left(-t^{\prime}\right)=\overline{\mathfrak{T}}_{0}^{\prime B}\left(t^{\prime}\right), \quad \mathfrak{T}_{-2}^{B}\left(-t^{\prime}\right) & =\mathfrak{T}_{1}^{\prime}{ }^{B}\left(t^{\prime}\right), \quad \mathfrak{T}_{-1}^{B}\left(-t^{\prime}\right)=\mathfrak{T}_{0}^{\prime B}\left(t^{\prime}\right), \\
\bar{x}_{1}=-\bar{x}_{0}^{\prime}, \quad \bar{x}_{-1} & =-\bar{x}_{2}^{\prime}, \quad \xi=-\xi^{\prime},
\end{aligned}
$$

из уже доказанного соотношения (5.1),

$$
\overline{\mathfrak{T}}_{-2}^{B}\left(-t^{\prime}\right)=\mathfrak{T}_{-2}^{B}\left(-t^{\prime}\right)+\sin ^{-1}\left(\bar{x}_{1}-\xi\right) \sin ^{-1}\left(\bar{x}_{1}-\bar{x}_{-1}\right) \mathfrak{T}_{-1}^{B}\left(-t^{\prime}\right) \quad \forall t^{\prime} \in\left(\alpha^{\prime}, \beta^{\prime}\right),
$$

легко получаем тождество

$$
\overline{\mathfrak{T}}_{0}^{\prime B}\left(t^{\prime}\right)=\mathfrak{T}_{1}^{\prime B}\left(t^{\prime}\right)+\sin \left(\bar{x}_{0}^{\prime}-\xi^{\prime}\right) \sin ^{-1}\left(\bar{x}_{0}^{\prime}-\bar{x}_{2}^{\prime}\right) \mathfrak{T}_{0}^{\prime B}\left(t^{\prime}\right) \quad \forall t^{\prime} \in\left(\alpha^{\prime}, \beta^{\prime}\right)
$$

последнее с точностью до обозначений совпадает с тождеством (5.3), что и требовалось.

3. Для доказательства теоремы осталось установить справедливость тождества $(5.2)$, для чего достаточно доказать его на отрезках $\left[x_{-1}, x_{0}\right],\left[x_{0}, x_{1}\right],\left[x_{1}, x_{2}\right],\left[x_{2}, x_{3}\right]$. Поскольку здесь рассуждения аналогичны первому пункту доказательства теоремы, то подробности будем опускать.

3.1. При $t \in\left[x_{-1}, x_{0}\right]$ согласно формуле (1.3) левая часть в (5.2) имеет вид

$$
\overline{\mathfrak{T}}_{-1}^{B}(t)=\sin ^{2}\left(t-\bar{x}_{-1}\right) \sin ^{-1}\left(\bar{x}_{1}-\bar{x}_{-1}\right) \sin ^{-1}\left(\bar{x}_{0}-\bar{x}_{-1}\right)
$$

и получается из первого слагаемого правой части

$$
\mathfrak{T}_{-1}^{B}(t)=\sin ^{2}\left(t-\bar{x}_{-1}\right) \sin ^{-1}\left(\xi-\bar{x}_{-1}\right) \sin ^{-1}\left(\bar{x}_{0}-\bar{x}_{-1}\right)
$$

умножением на выражение $\sin \left(\xi-\bar{x}_{-1}\right) \sin ^{-1}\left(\bar{x}_{1}-\bar{x}_{-1}\right)$. Поскольку второе слагаемое на рассматриваемом промежутке равно нулю, то при $t \in\left[x_{-1}, x_{0}\right]$ тождество (5.2) доказано.

3.2. При $t \in\left[x_{0}, x_{1}\right]$ левая часть соотношения (5.2) принимает вид

$$
\begin{aligned}
\overline{\mathfrak{T}}_{-1}^{B}(t)= & \frac{1}{2}\left[\sin \left(\bar{x}_{1}+\bar{x}_{2}-2 t\right) \cos \left(\bar{x}_{0}-\bar{x}_{-1}\right)+\sin \left(2 t-\bar{x}_{-1}-\bar{x}_{0}\right) \cos \left(\bar{x}_{2}-\bar{x}_{1}\right)\right. \\
& \left.+\sin \left(\bar{x}_{0}+\bar{x}_{-1}-\bar{x}_{1}-\bar{x}_{2}\right)\right] \sin ^{-1}\left(\bar{x}_{1}-\bar{x}_{0}\right) \sin ^{-1}\left(\bar{x}_{2}-\bar{x}_{0}\right) \sin ^{-1}\left(\bar{x}_{1}-\bar{x}_{-1}\right),
\end{aligned}
$$

а функции $\mathfrak{T}_{-1}^{B}$ и $\mathfrak{T}_{0}^{B}$ в правой части (согласно формулам (1.4) и (1.3) соответственно и с учетом обозначений (3.1)) могут быть записаны в форме:

$$
\begin{gathered}
\mathfrak{T}_{-1}^{B}(t)=\frac{1}{2}\left[\sin \left(\xi+\bar{x}_{1}-2 t\right) \cos \left(\bar{x}_{0}-\bar{x}_{-1}\right)+\sin \left(2 t-\bar{x}_{-1}-\bar{x}_{0}\right) \cos \left(\bar{x}_{1}-\xi\right)\right. \\
\left.+\sin \left(\bar{x}_{0}+\bar{x}_{-1}-\xi-\bar{x}_{1}\right)\right] \sin ^{-1}\left(\xi-\bar{x}_{0}\right) \sin ^{-1}\left(\bar{x}_{1}-\bar{x}_{0}\right) \sin ^{-1}\left(\xi-\bar{x}_{-1}\right)
\end{gathered}
$$

И

$$
\mathfrak{T}_{0}^{B}(t)=\sin ^{2}\left(t-\bar{x}_{0}\right) \sin ^{-1}\left(\bar{x}_{1}-\bar{x}_{0}\right) \sin ^{-1}\left(\xi-\bar{x}_{0}\right) .
$$


Таким образом, требуется установить тождество

$$
\begin{aligned}
& {\left[\sin \left(\bar{x}_{1}+\bar{x}_{2}-2 t\right) \cos \left(\bar{x}_{0}-\bar{x}_{-1}\right)+\sin \left(2 t-\bar{x}_{-1}-\bar{x}_{0}\right) \cos \left(\bar{x}_{2}-\bar{x}_{1}\right)\right.} \\
& \left.\quad+\sin \left(\bar{x}_{0}+\bar{x}_{-1}-\bar{x}_{1}-\bar{x}_{2}\right)\right] \sin ^{-1}\left(\bar{x}_{1}-\bar{x}_{0}\right) \sin ^{-1}\left(\bar{x}_{2}-\bar{x}_{0}\right) \sin ^{-1}\left(\bar{x}_{1}-\bar{x}_{-1}\right) \\
& \stackrel{?}{\equiv} \sin \left(\xi-\bar{x}_{-1}\right) \sin ^{-1}\left(\bar{x}_{1}-\bar{x}_{-1}\right)\left[\sin \left(\xi+\bar{x}_{1}-2 t\right) \cos \left(\bar{x}_{0}-\bar{x}_{-1}\right)\right. \\
& \left.\quad+\sin \left(2 t-\bar{x}_{-1}-\bar{x}_{0}\right) \cos \left(\bar{x}_{1}-\xi\right)+\sin \left(\bar{x}_{0}+\bar{x}_{-1}-\xi-\bar{x}_{1}\right)\right] \sin ^{-1}\left(\xi-\bar{x}_{0}\right) \\
& \quad \times \sin ^{-1}\left(\bar{x}_{1}-\bar{x}_{0}\right) \sin ^{-1}\left(\xi-\bar{x}_{-1}\right) \\
& \quad+2 \sin \left(\bar{x}_{2}-\xi\right) \sin ^{-1}\left(\bar{x}_{2}-\bar{x}_{0}\right) \sin ^{2}\left(t-\bar{x}_{0}\right) \sin ^{-1}\left(\bar{x}_{1}-\bar{x}_{0}\right) \sin ^{-1}\left(\xi-\bar{x}_{0}\right)
\end{aligned}
$$

котороепосле умножения на произведение $\sin \left(\bar{x}_{1}-\bar{x}_{-1}\right) \sin \left(\bar{x}_{1}-\bar{x}_{0}\right) \sin \left(\bar{x}_{2}-\bar{x}_{0}\right) \sin \left(\xi-\bar{x}_{0}\right)$ принимает вид

$$
\begin{aligned}
& \sin \left(\xi-\bar{x}_{0}\right)\left[\sin \left(\bar{x}_{1}+\bar{x}_{2}-2 t\right) \cos \left(\bar{x}_{0}-\bar{x}_{-1}\right)+\sin \left(2 t-\bar{x}_{-1}-\bar{x}_{0}\right) \cos \left(\bar{x}_{2}-\bar{x}_{1}\right)\right. \\
& \left.\quad+\sin \left(\bar{x}_{0}+\bar{x}_{-1}-\bar{x}_{1}-\bar{x}_{2}\right)\right] \stackrel{?}{=} \sin \left(\bar{x}_{2}-\bar{x}_{0}\right)\left[\sin \left(\xi+\bar{x}_{1}-2 t\right) \cos \left(\bar{x}_{0}-\bar{x}_{-1}\right)\right. \\
& \left.\quad+\cos \left(\bar{x}_{1}-\xi\right) \times \sin \left(2 t-\bar{x}_{-1}-\bar{x}_{0}\right)+\sin \left(\bar{x}_{0}+\bar{x}_{-1}-\xi-\bar{x}_{1}\right)\right] \\
& \quad+2 \sin \left(\bar{x}_{1}-\bar{x}_{-1}\right) \sin ^{2}\left(t-\bar{x}_{0}\right) \sin \left(\bar{x}_{2}-\xi\right)
\end{aligned}
$$

Полагая $a \stackrel{\text { def }}{=} \bar{x}_{-1}-t, b \stackrel{\text { def }}{=} \bar{x}_{0}-t, c \stackrel{\text { def }}{=} \bar{x}_{1}-t, d \stackrel{\text { def }}{=} \xi-t, e \stackrel{\text { def }}{=} \bar{x}_{2}-t$ в тождестве (4.1), приходим к соотношению (5.16). Таким образом, на промежутке $\left[x_{0}, x_{1}\right]$ тождество (5.2) справедливо.

3.3. При $t \in\left[x_{1}, x_{2}\right]$ в левой части соотношения (5.2) воспользуемся формулой (5.15), а в правой части функции $\mathfrak{T}_{-1}^{B}$ и $\mathfrak{T}_{0}^{B}$ (в силу формул (1.5) и (1.4) соответственно с учетом обозначений (3.1)) запишем в виде

$$
\mathfrak{T}_{-1}^{B}(t)=\sin ^{2}\left(t-\bar{x}_{1}\right) \sin ^{-1}\left(\bar{x}_{1}-\bar{x}_{0}\right) \sin ^{-1}\left(\bar{x}_{1}-\xi\right)
$$

и

$$
\begin{array}{r}
\mathfrak{T}_{0}^{B}(t)=\frac{1}{2}\left[\sin \left(\bar{x}_{1}+\bar{x}_{2}-2 t\right) \cos \left(\xi-\bar{x}_{0}\right)+\sin \left(2 t-\bar{x}_{0}-\xi\right) \cos \left(\bar{x}_{2}-\bar{x}_{1}\right)\right. \\
\left.+\sin \left(\xi+\bar{x}_{0}-\bar{x}_{1}-\bar{x}_{2}\right)\right] \sin ^{-1}\left(\bar{x}_{1}-\xi\right) \sin ^{-1}\left(\bar{x}_{2}-\xi\right) \sin ^{-1}\left(\bar{x}_{1}-\bar{x}_{0}\right)
\end{array}
$$

Итак, требуется доказать тождество

$$
\begin{aligned}
& {\left[\sin \left(\bar{x}_{1}+\bar{x}_{2}-2 t\right) \cos \left(\bar{x}_{0}-\bar{x}_{-1}\right)+\sin \left(2 t-\bar{x}_{-1}-\bar{x}_{0}\right) \cos \left(\bar{x}_{2}-\bar{x}_{1}\right)\right.} \\
& \left.\quad+\sin \left(\bar{x}_{0}+\bar{x}_{-1}-\bar{x}_{1}-\bar{x}_{2}\right)\right] \sin ^{-1}\left(\bar{x}_{1}-\bar{x}_{0}\right) \sin ^{-1}\left(\bar{x}_{2}-\bar{x}_{0}\right) \sin ^{-1}\left(\bar{x}_{1}-\bar{x}_{-1}\right) \\
& \stackrel{?}{=} 2 \sin (\xi-\bar{x}-1) \sin ^{-1}\left(\bar{x}_{1}-\bar{x}_{-1}\right) \sin ^{2}\left(t-\bar{x}_{1}\right) \sin ^{-1}\left(\bar{x}_{1}-\bar{x}_{0}\right) \sin ^{-1}\left(\bar{x}_{1}-\xi\right) \\
& \quad+\sin \left(\bar{x}_{2}-\xi\right) \sin ^{-1}\left(\bar{x}_{2}-\bar{x}_{0}\right)\left[\sin \left(\bar{x}_{1}+\bar{x}_{2}-2 t\right) \cos \left(\xi-\bar{x}_{0}\right)\right. \\
& \left.\quad+\sin \left(2 t-\bar{x}_{0}-\xi\right) \cos \left(\bar{x}_{2}-\bar{x}_{1}\right)+\sin \left(\xi+\bar{x}_{0}-\bar{x}_{1}-\bar{x}_{2}\right)\right] \\
& \quad \times \sin ^{-1}\left(\bar{x}_{1}-\xi\right) \sin ^{-1}\left(\bar{x}_{2}-\xi\right) \sin ^{-1}\left(\bar{x}_{1}-\bar{x}_{0}\right)
\end{aligned}
$$


которому умножением на произведение $\sin \left(\bar{x}_{1}-\bar{x}_{0}\right) \sin \left(\bar{x}_{2}-\bar{x}_{0}\right) \sin \left(\bar{x}_{1}-\bar{x}_{-1}\right) \sin \left(\bar{x}_{1}-\xi\right)$ легко придать вид

$$
\begin{aligned}
& \sin \left(\bar{x}_{1}-\xi\right)\left[\sin \left(\bar{x}_{1}+\bar{x}_{2}-2 t\right) \cos \left(\bar{x}_{0}-\bar{x}_{-1}\right)+\sin \left(2 t-\bar{x}_{-1}-\bar{x}_{0}\right) \cos \left(\bar{x}_{2}-\bar{x}_{1}\right)\right. \\
& \left.\quad+\sin \left(\bar{x}_{0}+\bar{x}_{-1}-\bar{x}_{1}-\bar{x}_{2}\right)\right] \stackrel{?}{=} 2 \sin \left(\bar{x}_{2}-\bar{x}_{0}\right) \sin \left(\xi-\bar{x}_{-1}\right) \sin ^{2}\left(t-\bar{x}_{1}\right) \\
& \quad+\sin \left(\bar{x}_{1}-\bar{x}_{-1}\right)\left[\sin \left(\bar{x}_{1}+\bar{x}_{2}-2 t\right) \cos \left(\xi-\bar{x}_{0}\right)+\sin \left(2 t-\bar{x}_{0}-\xi\right) \cos \left(\bar{x}_{2}-\bar{x}_{1}\right)\right. \\
& \left.\quad+\sin \left(\xi+\bar{x}_{0}-\bar{x}_{1}-\bar{x}_{2}\right)\right] .
\end{aligned}
$$

Снова воспользуемся леммой 1: полагая в тождестве (4.1) $a \stackrel{\text { def }}{=} \bar{x}_{2}-t, b \stackrel{\text { def }}{=} \bar{x}_{1}-t$, $c \stackrel{\text { def }}{=} \bar{x}_{0}-t, d \stackrel{\text { def }}{=} \xi-t, e \stackrel{\text { def }}{=} \bar{x}_{-1}-t$, придем к соотношению (5.17). Ввиду эквивалентности проведенных преобразований ясно, что тождество (5.2) справедливо на отрезке $t \in\left[x_{1}, x_{2}\right]$.

3.4. Обратимся, наконец, к случаю $t \in\left[x_{2}, x_{3}\right]$. Здесь левая часть в $(5.2)$ равна

$$
\overline{\mathfrak{T}}_{-1}^{B}(t)=\sin ^{2}\left(t-\bar{x}_{2}\right) \sin ^{-1}\left(\bar{x}_{2}-\bar{x}_{0}\right) \sin ^{-1}\left(\bar{x}_{2}-\bar{x}_{1}\right) .
$$

Она получается из функции

$$
\mathfrak{T}_{0}^{B}(t)=\sin ^{2}\left(t-\bar{x}_{2}\right) \sin ^{-1}\left(\bar{x}_{2}-\xi\right) \sin ^{-1}\left(\bar{x}_{2}-\bar{x}_{1}\right)
$$

умножением на выражение $\sin \left(\bar{x}_{2}-\xi\right) \sin ^{-1}\left(\bar{x}_{2}-\bar{x}_{0}\right)$; последнее с учетом равенства нулю первого слагаемого правой части (5.2) на рассматриваемом промежутке доказывает тождество (5.2) для $t \in\left[x_{2}, x_{3}\right]$.

На этом заканчивается рассмотрение третьего завершающего пункта доказательства. Теорема полностью доказана.

В дальнейшем рассматриваются бесконечные ряды вида $\sum_{j} c_{j} \mathfrak{T}_{j}^{B}, c_{j} \in \mathbb{R}^{1}$, где суммирование по $j$ распространено на все целые числа, $j \in \mathbb{Z}$. Заметим, что при каждом фиксированном $t \in(\alpha, \beta)$ такой ряд содержит не более трех ненулевых слагаемых; поэтому при любой последовательности $\left\{c_{j}\right\}_{j \in \mathbb{Z}}$ коэффициентов $c_{j} \in \mathbb{R}^{1}$ упомянутый ряд сходится (в смысле поточечной сходимости).

Обозначим $\mathscr{T}^{B}(X)$ линейное пространство, являющееся линейной оболочкой функций $\mathfrak{T}_{j}^{B}, \mathscr{T}^{B}(X) \stackrel{\text { def }}{=}\left\{\tilde{u} \mid \tilde{u} \stackrel{\text { def }}{=} \sum_{j} c_{j} \mathfrak{T}_{j}^{B} \forall c_{j} \in \mathbb{R}^{1}\right\} ;$ пространство $\mathscr{T}^{B}(X)$ будем назьвать пространством $B_{c s}$-сплайнов на сетке $X$, а функции $\mathfrak{T}_{j}^{B}-$ образующими $B_{c s}$-сплайнами (или просто - образующими) этого пространства.

В соответствии с этим определением $\mathscr{T}^{B}\left(\bar{X}_{1}\right)$ является пространством $B_{c s}$-сплайнов на сетке $\bar{X}_{1}$,

$$
\mathscr{T}^{B}\left(\bar{X}_{1}\right)=\left\{\tilde{u} \mid \tilde{u} \stackrel{\text { def }}{=} \sum_{j} \bar{c}_{j} \overline{\mathfrak{T}}_{j}^{B} \forall \bar{c}_{j} \in \mathbb{R}^{1}\right\} .
$$

ТЕОРема 3. Справедливо соотношение

$$
\overline{\mathfrak{T}}_{i}^{B}=\sum_{j} d_{i, j} \mathfrak{T}_{j}^{B}
$$


где при $i, j \in \mathbb{Z}$ коэффициенты $d_{i, j}$ определяются равенствами

$$
\begin{aligned}
& d_{i, j}=\delta_{i, j} \text { npu } i \leqslant-3 \text {, } \\
& d_{-2,-2}=1, \quad d_{-2,-1}=\frac{\sin \left(\bar{x}_{1}-\xi\right)}{\sin \left(\bar{x}_{1}-\bar{x}_{-1}\right)}, \quad d_{-2, j}=0 \text { nрu } j \notin\{-2,-1\} \text {, } \\
& d_{-1,-1}=\frac{\sin \left(\xi-\bar{x}_{-1}\right)}{\sin \left(\bar{x}_{1}-\bar{x}_{-1}\right)}, \quad d_{-1,0}=\frac{\sin \left(\bar{x}_{2}-\xi\right)}{\sin \left(\bar{x}_{2}-\bar{x}_{0}\right)}, \quad d_{-1, j}=0 \quad \text { npu } j \notin\{-1,0\}, \\
& d_{0,0}=\frac{\sin \left(\xi-\bar{x}_{0}\right)}{\sin \left(\bar{x}_{2}-\bar{x}_{0}\right)}, \quad d_{0,1}=1, \quad d_{0, j}=0 \quad \text { npu } j \notin\{0,1\}, \\
& d_{i, j}=\delta_{i+1, j} \text { npu } i \geqslant 1 \text {. }
\end{aligned}
$$

ДокАЗАТЕЛЬСТво непосредственно вытекает из формул (5.1)-(5.3) теоремы 2.

СлЕДСТВИЕ 1. Пространство $\mathscr{T}^{B}\left(\bar{X}_{1}\right)$ содерэится в пространстве $\mathscr{T}^{B}(X)$.

Соотношения (5.18) назьваются калибровочными соотношениями, функции $\mathfrak{T}_{j}^{B}$ на-

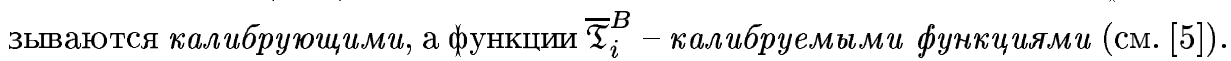

6. Всплесковое разложение. Формулы реконструкции. Рассмотрим оператор $P$ проектирования пространства $\mathscr{T}^{B}(X)$ на подпространство $\mathscr{T}^{B}\left(\bar{X}_{1}\right)$, задаваемьй формулой $P \tilde{u} \stackrel{\text { def }}{=} \sum_{j}\left\langle\bar{g}^{(j)}, \tilde{u}\right\rangle \overline{\mathfrak{T}}_{j}^{B} \forall \tilde{u} \in \mathscr{T}^{B}(X)$, и введем оператор $Q=I-P$, где $I-$ тождественный оператор.

В результатеполучаем пространство всплесков (вейвлетов) $W \stackrel{\text { def }}{=} Q \mathscr{T}^{B}(X)$ и прямое разложение

$$
\mathscr{T}^{B}(X)=\mathscr{T}^{B}(\bar{X}) \dot{+} W,
$$

которое называется сплайн-всплесковым разложениемпространства $\mathscr{T}^{B}(X)$.

Пусть известны коэффициенты $a_{i}$ и $b_{i^{\prime}}$ в разложениях проекций элемента $\tilde{u} \in \mathscr{T}^{B}(X)$ на пространства $\mathscr{T}^{B}(\bar{X})$ и $W: P \tilde{u}=\sum_{i} a_{i} \overline{\mathfrak{T}}_{i}^{B}, Q \tilde{u}=\sum_{i^{\prime}} b_{i^{\prime}} \mathfrak{T}_{i^{\prime}}^{B}$, где $a_{i}=\left\langle\bar{g}^{(i)}, \tilde{u}\right\rangle$, $b_{i^{\prime}}=\left\langle g^{\left(i^{\prime}\right)}, \tilde{u}\right\rangle$. В соответствии с формулой (5.18) имеем

$$
\tilde{u}=\sum_{i} a_{i} \overline{\mathfrak{T}}_{i}^{B}+\sum_{i^{\prime}} b_{i^{\prime}} \mathfrak{T}_{i^{\prime}}^{B}=\sum_{i^{\prime}}\left(\sum_{j} a_{i} d_{i, i^{\prime}}+b_{i^{\prime}}\right) \mathfrak{T}_{i^{\prime}}^{B},
$$

так что для чисел $c_{j}=\left\langle g^{(j)}, \tilde{u}\right\rangle$ получаем формуль реконструкиии

$$
c_{j}=\sum_{i} a_{i} d_{i, j}+b_{j}, \quad j \in \mathbb{Z} .
$$

ТЕоремА 4. Для рассматриваемого сплайн-всплескового разложсения (6.1) пространства $\mathscr{T}^{B}(X)$ формуль реконструкиии (6.2) имеют вид

$$
\begin{aligned}
c_{j} & =a_{j}+b_{j} \quad \text { npu } \quad j \leqslant-2, \\
c_{-1} & =a_{-2} \frac{\sin \left(\bar{x}_{1}-\xi\right)}{\sin \left(\bar{x}_{1}-\bar{x}-1\right)}+a_{-1} \frac{\sin \left(\xi-\bar{x}_{-1}\right)}{\sin \left(\bar{x}_{1}-\bar{x}_{-1}\right)}+b_{-1}, \\
c_{0} & =a_{-1} \frac{\sin \left(\bar{x}_{2}-\xi\right)}{\sin \left(\bar{x}_{2}-\bar{x}_{0}\right)}+a_{0} \frac{\sin \left(\xi-\bar{x}_{0}\right)}{\sin \left(\bar{x}_{2}-\bar{x}_{0}\right)}+b_{0}, \\
c_{j} & =a_{j-1}+b_{j} \quad \text { npu } \quad j \geqslant 1 .
\end{aligned}
$$


ДокАЗАТЕЛЬСтво. Ввиду равенств $(5.19),(5.20)$ при $j \leqslant-2$ имеем $c_{j}=a_{j} d_{j, j}+b_{j}$, $d_{j, j}=1$, а это эквивалентно формуле (6.3). При $j=-1$ находим $c_{-1}=a_{-2} d_{-2,-1}+$ $a_{-1} d_{-1,-1}+b_{-1}$ и из формул $(5.20),(5.21)$ получаем (6.4). При $j=-1$ имеем $c_{0}=$ $a_{-1} d_{-1,0}+a_{0} d_{0,0}+b_{0}$, откуда с помощю соотношений $(5.21),(5.22)$ вьводим (6.5). Наконец, при $j \geqslant 1$ пишем $c_{j}=a_{j-1} d_{j-1, j}+b_{j}, d_{j-1, j}=1$, так что с учетом формул (5.22), (5.23) получаем соотношение (6.6). Теорема доказана.

7. Формулы декомпозиции. Пусть теперь известны коэффициенты $c_{k}$ в разложении элемента $\tilde{u} \in \mathscr{T}^{B}(X)$ по элементам базиса $\mathfrak{T}_{i^{\prime}}^{B}$, а именно, $\tilde{u}=\sum_{k} c_{k} \mathfrak{T}_{k}^{B}$. Используя равенство $a_{i}=\left\langle\bar{g}^{(i)}, \tilde{u}\right\rangle$, последовательно имеем

$$
b_{j}=c_{j}-\sum_{i} d_{i, j} a_{i}=c_{j}-\sum_{i} d_{i, j}\left\langle\bar{g}^{(i)}, \sum_{k} c_{k} \mathfrak{T}_{k}^{B}\right\rangle=c_{j}-\sum_{i} d_{i, j} \sum_{k} c_{k}\left\langle\bar{g}^{(i)}, \mathfrak{T}_{k}^{B}\right\rangle .
$$

Отсюда получаются формуль декомпозиции

$$
\begin{aligned}
& a_{i}=\sum_{k} c_{k}\left\langle\bar{g}^{(i)}, \mathfrak{T}_{k}^{B}\right\rangle \\
& b_{j}=c_{j}-\sum_{i} d_{i, j} \sum_{k} c_{k}\left\langle\bar{g}^{(i)}, \mathfrak{T}_{k}^{B}\right\rangle .
\end{aligned}
$$

ТЕОремА 5. Выражсения $\left\langle\bar{g}^{(i)}, \mathfrak{T}_{j}^{B}\right\rangle, i, j \in \mathbb{Z}$, отличны от нуля только в следующих случаях:

$$
\begin{aligned}
\left\langle\bar{g}^{(i)}, \mathfrak{T}_{i}^{B}\right\rangle & =1 \quad \text { npu } \quad i \leqslant-2, \\
\left\langle\bar{g}^{(i)}, \mathfrak{T}_{i+1}^{B}\right\rangle & =1 \quad \text { npu } \quad i \geqslant 0, \\
\left\langle\bar{g}^{(-1)}, \mathfrak{T}_{-2}^{B}\right\rangle & =-\frac{\sin \left(\bar{x}_{1}-\xi\right)}{\sin \left(\xi-\bar{x}_{-1}\right)}, \\
\left\langle\bar{g}^{(-1)}, \mathfrak{T}_{-1}^{B}\right\rangle & =\frac{\sin \left(\bar{x}_{1}-\bar{x}_{-1}\right)}{\sin \left(\xi-\bar{x}_{-1}\right)}
\end{aligned}
$$

в остальных случаях вырахсение $\left\langle\bar{g}^{(i)}, \mathfrak{T}_{j}^{B}\right\rangle$ равно нулю, т.е.

$$
\begin{gathered}
\left\langle\bar{g}^{(i)}, \mathfrak{T}_{j}^{B}\right\rangle=0 \\
n p u \quad(i, j) \notin\{(i, i) \mid i \leqslant-2\} \cup\{(i, i+1) \mid i \geqslant 0\} \cup\{(-1,-2),(-1,-1)\} .
\end{gathered}
$$

ДокАЗАТЕЛЬСТво. Из формул (3.2) с учетом свойства биортогональности (см. теорему 1) следует, что

$$
\begin{array}{ll}
\left\langle\bar{g}^{(i)}, \mathfrak{T}_{j}^{B}\right\rangle=\left\langle\bar{g}^{(i)}, \overline{\mathfrak{T}}_{j}^{B}\right\rangle=\delta_{i, j} & \text { при } j \leqslant-3, \\
\left\langle\bar{g}^{(i)}, \mathfrak{T}_{j}^{B}\right\rangle=\left\langle\bar{g}^{(i)}, \overline{\mathfrak{T}}_{j-1}^{B}\right\rangle=\delta_{i, j-1} & \text { при } j \geqslant 1,
\end{array}
$$

а из формул (3.3) аналогично вытекает, что

$$
\begin{array}{ll}
\left\langle\bar{g}^{(i)}, \mathfrak{T}_{j}^{B}\right\rangle=\left\langle g^{(i)}, \mathfrak{T}_{j}^{B}\right\rangle=\delta_{i, j} & \text { при } i \leqslant-2, \\
\left\langle\bar{g}^{(i)}, \mathfrak{T}_{j}^{B}\right\rangle=\left\langle g^{(i+1)}, \mathfrak{T}_{j}^{B}\right\rangle=\delta_{i+1, j} & \text { при } i \geqslant 0 .
\end{array}
$$


Осталось найти лишь три величины $\left\langle\bar{g}^{(-1)}, \mathfrak{T}_{0}^{B}\right\rangle,\left\langle\bar{g}^{(-1)}, \mathfrak{T}_{-1}^{B}\right\rangle$ и $\left\langle\bar{g}^{(-1)}, \mathfrak{T}_{-2}^{B}\right\rangle$. Поскольку $\operatorname{supp} \bar{g}^{(-1)}=\bar{x}_{0}$, первая величина равна нулю, $\left\langle\bar{g}^{(-1)}, \mathfrak{T}_{0}^{B}\right\rangle=0$; отсюда, учитывая формулы (7.9) - (7.12), заключаем, что соотношения (7.3), (7.4) и (7.7), (7.8) доказаны. Для второй величины имеем

$$
\begin{aligned}
& \left\langle\bar{g}^{(-1)}, \mathfrak{T}_{-1}^{B}\right\rangle=\left\langle\cos \left(\bar{x}_{1}-\bar{x}_{0}\right)+\left.\frac{1}{2} \sin \left(\bar{x}_{1}-\bar{x}_{0}\right) \frac{d}{d t}\right|_{t=\bar{x}_{1}}, \sin ^{2}\left(t-\bar{x}_{-1}\right)\right\rangle \\
& \times \sin ^{-1}\left(\xi-\bar{x}_{-1}\right) \sin ^{-1}\left(\bar{x}_{0}-\bar{x}_{-1}\right) \\
& =\sin \left(\bar{x}_{1}-\bar{x}_{-1}\right) \sin ^{-1}\left(\xi-\bar{x}_{-1}\right) \text {; }
\end{aligned}
$$

отсюда следует соотношение (7.6).

Аналогично устанавливается формула (7.5):

$$
\begin{aligned}
\left\langle\bar{g}^{(-1)}, \mathfrak{T}_{-2}^{B}\right\rangle= & \left\langle\cos \left(\bar{x}_{1}-\bar{x}_{0}\right)+\left.\frac{1}{2} \sin \left(\bar{x}_{1}-\bar{x}_{0}\right) \frac{d}{d t}\right|_{t=\bar{x}_{1}}, \sin ^{2}(t-\xi)\right\rangle \\
& \times \sin ^{-1}\left(\xi-\bar{x}_{-1}\right) \sin ^{-1}\left(\xi-\bar{x}_{0}\right)=-\sin \left(\bar{x}_{1}-\xi\right) \sin ^{-1}\left(\xi-\bar{x}_{-1}\right) .
\end{aligned}
$$

Теорема полностью доказана.

Теорема 6. Для сплайн-всплескового разложения (6.1) пространства $\mathscr{T}^{B}(X)$ формуль декомпозичии (7.1) имеют форму

$$
\begin{gathered}
a_{i}=c_{i} \quad \text { npu } \quad i \leqslant-2 \\
a_{-1}=-\sin \left(\bar{x}_{1}-\xi\right) \sin ^{-1}\left(\xi-\bar{x}_{-1}\right) c_{-2}+\sin \left(\bar{x}_{1}-\bar{x}_{-1}\right) \sin ^{-1}\left(\xi-\bar{x}_{-1}\right) c_{-1} \\
a_{i}=c_{i+1} \quad \text { npu } \quad i \geqslant 0
\end{gathered}
$$

а формуль (7.2) принимают вид

$$
\begin{gathered}
b_{j}=0 \quad \text { npu } \quad j \neq 0, \\
b_{0}=c_{0}+\left[\sin \left(\bar{x}_{2}-\xi\right) \sin \left(\bar{x}_{1}-\xi\right) c_{-2}-\left(\sin \left(\bar{x}_{2}-\xi\right) \sin \left(\bar{x}_{1}-\bar{x}_{-1}\right)\right.\right. \\
\left.\left.+\sin \left(\xi-\bar{x}_{0}\right) \sin \left(\xi-\bar{x}_{-1}\right)\right) c_{1}\right] \sin ^{-1}\left(\bar{x}_{2}-\bar{x}_{0}\right) \sin ^{-1}\left(\xi-\bar{x}_{-1}\right) .
\end{gathered}
$$

ДокАЗАТЕльСтво. Начнем с доказательства формул (7.15)-(7.17). Если $i \neq-1$, то согласно теореме 5 в соотношении (7.1) отлично от нуля разве лишь одно слагаемое: при $i \leqslant-2$ это слагаемое имеет индекс $k=i$, а при $i \geqslant 0$ это слагаемое с индексом $k=i+1$. Принимая во внимание равенства (7.3) и (7.4) соответственно, приходим к формулам (7.15) и (7.17). Обрашаясь к доказательству формулы (7.16) возьмем $i=-1$ в соотношении (7.1) и воспользуемся теоремой 5 ; используя равенства (7.5)-(7.8) получаем формулу (7.16). 
Для доказательства соотношений (7.18), (7.19) воспользуемся равенствами (6.3)(6.6): с помощью (7.15) и (6.3) находим $b_{j}=0$ при $j \leqslant-2$, и благодаря (7.17) и (6.6) получаем $b_{j}=0$ при $j \geqslant 1$, а учитьвая еше формулу (6.4), вьводим равенство

$$
\begin{aligned}
b_{-1}= & c_{-1}-c_{-2} \sin \left(\bar{x}_{1}-\xi\right) \sin ^{-1}\left(\bar{x}_{1}-\bar{x}_{-1}\right)-\left[-\sin \left(\bar{x}_{1}-\xi\right) \sin ^{-1}\left(\xi-\bar{x}_{-1}\right) c_{-2}\right. \\
& \left.+\sin \left(\bar{x}_{1}-\bar{x}_{-1}\right) \sin ^{-1}\left(\xi-\bar{x}_{-1}\right) c_{-1}\right] \sin \left(\xi-\bar{x}_{-1}\right) \sin \left(\bar{x}_{1}-\bar{x}_{-1}\right)=0
\end{aligned}
$$

все только что полученные равенства приводят к формуле (7.18).

Подставляя в равенство (6.5) величины $a_{-1}$ и $a_{0}$ из формул $(7.16)$ и $(7.17)$, находим соотношение (7.19).

Теорема доказана.

8. О добавлении произвольного числа узлов. Пусть $X_{0}$ - сетка вида (1.1) со свойством (1.2). Рассмотрим бесконечную расширяющуюся последовательность сеток $X_{0} \subset X_{1} \subset X_{2} \subset X_{3} \subset \cdots$, каждая из которых отличается от предыдущей добавлением одного узла, не совпадающего с узлами расширяемой сетки. Соответствующая последовательность пространств $\mathscr{T}^{B}\left(X_{s}\right) B_{c s}$-сплайнов обладает свойством вложенности $\mathscr{T}^{B}\left(X_{s}\right) \subset \mathscr{T}^{B}\left(X_{s+1}\right), s=0,1,2, \ldots$ (см. следствие 1 при $\left.\bar{X}_{1}=X_{s}, X=X_{s+1}\right)$, так что $\mathscr{T}^{B}\left(X_{0}\right) \subset \mathscr{T}^{B}\left(X_{1}\right) \subset \mathscr{T}^{B}\left(X_{2}\right) \subset \cdots$. Обозначая через $\mathfrak{T}_{i(s)}^{B}, i \in \mathbb{Z}$, образующие $B_{c s}$-сплайны пространства $\mathscr{T}^{B}\left(X_{s}\right)$, видим, что справедливы калибровочные соотношения

$$
\mathfrak{T}_{i(s)}^{B}=\sum_{j} d_{i, j}^{(s)} \mathfrak{T}_{i(s+1)}^{B},
$$

где коэффициенты $d_{i, j}^{(s)}$ определяются согласно теореме 3 (в которой следует взять $\bar{X}_{1}=$ $X_{s}, X=X_{s+1}$ и произвести подходящую перенумерацию узлов). Заметим, что ввиду упомянутой теоремы в каждом из соотношений (8.1) имеется не более двух ненулевых слагаемых. Применяя к системе вложенных пространств предыдущие построения, получаем сплайн-всплесковое разложение вида $\mathscr{T}^{B}\left(X_{s+1}\right)=\mathscr{T}^{B}\left(X_{s}\right) \dot{+} W_{s}$, последовательное применение которого приводит к прямому разложению пространства $\mathscr{T}_{*}^{B} \stackrel{\text { def }}{=} \bigcup_{s} \mathscr{T}^{B}\left(X_{s}\right)$ в виде прямой суммы $\mathscr{T}_{*}^{B}=\mathscr{T}^{B}\left(X_{0}\right) \dot{+} W_{0}+W_{1} \dot{+} W_{2} \cdots$ и к формулам реконструкции и декомпозиции (см. формулы $(6.2),(7.1),(7.2)$ и теоремы 4,5$)$.

Из последовательности $0,1,2,3, \ldots$ выделим подпоследовательность $s_{0}<s_{1}<s_{2}<$ $\cdots<s_{p}<\cdots<s_{q}<\cdots$ и рассмотрим соответствующую последовательность сеток $X_{s_{0}} \subset X_{s_{1}} \subset X_{s_{2}} \subset \cdots$. Сетка $X_{s_{q}}$ получается из сетки $X_{s_{p}}$ добавлением $s_{q}-s_{p}$ узлов, и потому $\mathscr{T}^{B}\left(X_{s_{p}}\right) \subset \mathscr{T}^{B}\left(X_{s_{p}+1}\right) \subset \mathscr{T}^{B}\left(X_{s_{p}+2}\right) \subset \cdots \subset \mathscr{T}^{B}\left(X_{s_{q}}\right)$. Рекуррентно применяя соотношение (8.1) при $s=s_{p}, s_{p}+1, \ldots, s_{q}-1$, находим калибровочные соотношения

$$
\mathfrak{T}_{i\left(s_{p}\right)}^{B}=\sum_{j} d_{i, j}^{\left(s_{p}, s_{q}\right)} \mathfrak{T}_{j\left(s_{q}\right)}^{B},
$$

где функции $\mathfrak{T}_{i\left(s_{p}\right)}^{B}$ и $\mathfrak{T}_{i\left(s_{q}\right)}^{B}$ строятся по формулам (1.3)-(1.6) при $X=X_{s_{p}}$ и при $X=X_{s_{q}}$ соответственно. Нетрудно видеть, что число ненулевых слагаемых в соотношении (8.2) не превосходит числа $s_{q}-s_{p}$. При вычислении коэффициентов $d_{i, j}^{\left(s_{p}, s_{q}\right)}$ нет необходимости в упомянутом рекуррентном процессе: можно лишь применить систему функционалов, биортогональную к системе $\left\{\mathfrak{T}_{j\left(s_{q}\right)}^{B}\right\}_{j \in \mathbb{Z}}$. Упомянутая система функционалов $\left\{g^{(i)}\right\}_{i \in \mathbb{Z}}$ определяется формулами $(2.1)$ при $X=X_{s_{q}}$. Очевидно, что $d_{i, j}^{\left(s_{p}, s_{q}\right)}=$ 
$\left\langle g^{(i)}, \mathfrak{T}_{j\left(s_{p}\right)}^{B}\right\rangle ;$ с помощью биортогональных систем в этом случае легко получаются формулы реконструкции и декомпозиции.

Предположим теперь, что объединение $\widetilde{X} \stackrel{\text { def }}{=} \bigcup_{s=0}^{+\infty} X_{s}$ представляет собой сетку вида (1.1) со свойством (1.2), так что между любыми двумя соседними узлами сетки $X_{0}$ имеется лишш конечное число узлов сетки $\tilde{X}$; при этом $X_{0} \subset \widetilde{X}$ и $\mathscr{T}^{B}\left(X_{0}\right) \subset \mathscr{T}^{B}(\widetilde{X})$. Отсюда следует, что справедливы калибровочные соотношения $\mathfrak{T}_{i}^{B}=\sum_{j} \tilde{d}_{i, j} \widetilde{\mathfrak{T}}_{j}^{B}$ между образуюшими $\mathfrak{T}_{i}^{B}$ и $\widetilde{\mathfrak{T}}_{j}^{B}$ пространств $\mathscr{T}^{B}\left(X_{0}\right)$ и $\mathscr{T}^{B}(\tilde{X})$ соответственно, причем число ненулевых слагаемых в каждом из них конечно. Как и прежде, биортогональные системы к упомянутым образуюшим определяются теми же формулами (2.1) при $X=X_{0}$ и при $X=\widetilde{X}$ соответственно. В частности, если $\left\{g^{(i)}\right\}_{i \in \mathbb{Z}}-$ биортогональная система функционалов к системе сплайнов $\left\{\widetilde{\mathfrak{T}}_{j}^{B}\right\}_{j \in \mathbb{Z}}$, то $\tilde{d}_{i, j}=\left\langle g^{(i)}, \mathfrak{T}_{j}^{B}\right\rangle$. Упомянутые биортогональные системы стандартным способом приводят к формулам реконструкции и декомпозиции (см. формулы (6.2) и (7.1), (7.2)).

\section{СПИСОК ЦИТИРОВАННОЙ ЛИТЕРАТУРЫ}

[1] Daubechies I. Ten Lectures on Wavelets. CMBS-NSR Regional Conference Series in Appl. Math. V. 61. Philadelphia, PA: SIAM, 1992.

[2] Jawerth B., Sweldens W. An overview of wavelet based multiresolution analyses // SIAM Rev. 1994. V. 36. P. 377-412.

[3] Новиков И.Я., Стечкин С.Б. Основы теории всплесков // УМН. 1998. Т. 53. №6. C. $53-128$.

[4] Демьянович Ю. К. Калибровочное соотношение для $B$-сплайнов на неравномерной сетке // Матем. моделирование. 2001. Т. 13. №9. С. 98-100.

[5] Демьянович Ю. К. Всплесковые разложения в пространствах сплайнов на неравномерной сетке // Докл. РАН. 2002. Т. 382. № 3. С. 313-316.

[6] Бахвалов Н. С. О сходимости одного релаксационного метода при естественных ограничениях на эллиптический оператор // ЖВМиМФ. 1966. Т. 6. № 5. С. 861-883.

[7] Завьялов Ю. С., Квасов Б. И., Мирошниченко В. Л. Методы сплайн-функций. М.: Наука, 1980.

Санкт-Петербургский государственный университет

Поступило

E-mail: Yuri.Demjanovich@paloma.spbu.ru

25.08.2004 